\title{
Concomitant Mycobacterium tuberculosis infection promotes lung tumor growth through enhancing Treg development
}

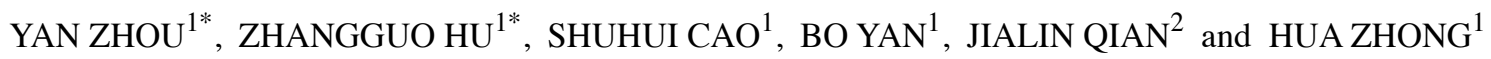 \\ Departments of ${ }^{1}$ Pulmonary Disease, and ${ }^{2}$ Respiration Medicine, Shanghai Chest Hospital, \\ Shanghai Jiao Tong University, Shanghai 200030, P.R. China
}

Received November 11, 2016; Accepted April 26, 2017

DOI: $10.3892 /$ or.2017.5733

\begin{abstract}
Lung cancer is the most common malignancy in humans. An increased population of $\mathrm{CD}^{+}{ }^{+} \mathrm{Foxp} 3^{+}$regulatory $\mathrm{T}$ cells (Tregs) in the tumor-associated microenvironment plays an important role in cancer immune evasion. The exact role and the involved mechanisms of concomitant $\mathrm{H} 37 \mathrm{Rv}$ infection in non-small cell lung cancer (NSCLC) development are still not clear. Here, we showed that H37Rv infection promoted NSCLC cell growth with a higher percentage of Tregs found in draining lymph nodes. We also determined in vitro that $\mathrm{H} 37 \mathrm{Rv}$ infection induced macrophage maturation and PD-L1 expression, which promoted Treg proportion, with enhanced proliferation suppression function. Mechanism analysis revealed that AKT-mTORC1 signal was important for PD-L1 expression induced by H37Rv infection. Suppressing of AKT-mTORC1 signal by rapamycin or raptor deficiency showed decreased PD-L1 levels which further reduced Treg proportion in a co-culture system. Finally, tumor-bearing mice injected with $\mathrm{H} 37 \mathrm{Rv}$ plus rapamycin enhance the immune response of lung cancer compared with injected with $\mathrm{H} 37 \mathrm{Rv}$ alone. This study demonstrated that concomitant H37Rv infection promote NSCLC tumor immune eacape through enhancing Treg proportion.
\end{abstract}

\section{Introduction}

Lung cancer is one of the most common malignancies with severe mortality worldwide $(1,2)$. There are ermerging

Correspondence to: Dr Hua Zhong, Department of Pulmonary Disease, Shanghai Chest Hospital, Shanghai Jiao Tong University, 241 Huaihai Road (W), Shanghai 200030, P.R. China

E-mail: zhonghuachina_vip@126.com

Dr Jialin Qian, Department of Respiration Medicine, Shanghai Chest Hospital, Shanghai Jiao Tong University, 241 Huaihai Road (W), Shanghai 200030, P.R. China

E-mail: jialin_qian@aliyun.com

*Contributed equally

Key words: Mycobacterium tuberculosis, infection, Treg, lung tumor, macrophages treatments for lung cancer including surgery, irradiation, chemotherapy and immunotherapy. However, prognosis remains unsatisfactory (3-5). Recently, comorbid cancer-infection which represents an independent concomitant microorganism infection in tumor has attracted new attention, since certain microorganism infection might induce antitumor immunity responses for a new treatment strategy. Malaria infection significantly suppresses murine Lewis lung cancer growth via induction of innate and adaptive antitumor responses in a mouse model, suggesting that the malaria parasite may stand for a new strategy or therapeutic vaccine vector for anti-lung cancer immunotherapy (6). T. gondii infection inhibits tumor growth in the Lewis lung carcinoma mouse model through the induction of Th1 immune responses and anti-angiogenic activity (7).

Mycobacterium tuberculosis (MTB) is an obligate pathogenic bacterial species in the family Mycobacteriaceae and the causative agent of tuberculosis (8). Mononuclear cells recruited to sites of MTB infection or novel MTB antigens, are exposed to MTB Toll-like receptor (TLR) ligands. MTB is rich in TLR2 ligands $(9,10)$, and a role for TLR2 ligand in expansion of Treg has been previously shown (11). MTB and its components expand functional CD $4^{+}$Foxp $3^{+}$Treg, which implicates for effective immunization against $\operatorname{MTB}(12,13)$. It was also reported that active tuberculosis in non-small cell lung cancer (NSCLC) patients shows better survival outcome, possibly due to the $\mathrm{T}$ lymphocyte infiltration in tumors (14). However, the role of an independent H37Rv infection in the development of NSCLC is not quite clear.

Here, we demonstrated that independent MTB H37Rv infection facilitated NSCLC progression. H37Rv cocommitant infection promoted Treg differentiation and its suppressive function through enhancing PD-L1 expression on macrophages. Mechanically, Akt-mTORC1 is responsible for H37Rv sitmulated PD-L1 expression on macrophages. Inactivation of mTORC1 by rapamycin or knockdown of raptor dereased Treg proportion and further reduced tumor development enhanced by H37Rv concomitant infection.

\section{Materials and methods}

Mice, cells, and bacteria. Female 8- to 10-week-old C57BL/6 mice were purchased from the SLAC Laboratory (Shanghai, China) and raised in the Animal Center of the Shanghai Chest 
Hospital. The animal experiment facilities were approved by the Shanghai Jiao Tong University School of Medicine Animal Care and Use Committee. All surgery was performed under anesthesia, and all efforts were made to minimize animal suffering. The murine LLC cell line was obtained from the Chinese Academy of Sciences Cell Bank (Shanghai, China). The H37Rv strain was obtained from the Shanghai Pulmonary Hospital as a gift.

Antibodies. Neutralizing antibody to PD-L1 and control IgG were obtained from BioXcell. Antibodies used in western blotting were all from Cell Signaling Technology.

Mouse models. C57BL/6 mice were s.c. injected with $2 \times 10^{6}$ murine LLC cells to establish tumors. At the same time, the tumor cell-inoculated mice were infected peritoneally with $2 \times 10^{6}$ heat-killed H37Rv (H37Rv), while challenged peritoneal with PBS were used as the control group (Ctr). Animals were examined daily until the tumors became palpable, after which the tumor volume was determined daily by measuring the diameter of the tumors using calipers. The volume was calculated using the formula, $\mathrm{V}=(\mathrm{ab} 2) / 2$, where a is the long axis, and $b$ is the short axis.

Rapamycin (Sigma) treatment was performed by injecting intraperitoneally with $4 \mathrm{mg} / \mathrm{kg}$ rapamycin or vehicle solution twice a week. Rapamycin was first dissolved in $100 \%$ ethanol at $10 \mathrm{mg} / \mathrm{ml}$, diluted in vehicle solution containing 5\% Tween-80 and 5\% PEG-400 in PBS to $0.5 \mathrm{mg} / \mathrm{ml}$, and filtered (15).

Flow cytometry. The following antibodies and their corresponding isotype controls (all purchased from eBioscience, USA) were used for staining: CD4-Percp, Foxp3-FITC, CD11c-FITC, CD80-PE, MHCII-PE, PD-L1-PE, F4/80-FITC. CFSE were obtained from Invitrogen, USA. Cells were washed, fixed and stained according to the manufacturer's instructions. Samples were run on a FACSCalibur (BD Biosciences) and analyzed using FlowJo software (TreeStar).

Quantitative RT-PCR. RNA was isolated from cells using the Qiagen RNeasy Mini kit (Qiagen). cDNA was made using the SuperScript II RT Reaction kit (Invitrogen) from $2 \mu \mathrm{g}$ of isolated RNA. Samples were analyzed on a ABI 9500 RT-PCR System Instrument using SYBR PCR Master Mix according to the manufacturer's instructions. Specific primers were as follows: Foxp3 forward, GGCCCTTCTCCAGGACAGA; reverse, GGCATGGGCATCCACAGT. T-bet forward, ACCT GTTGTGGTCCAAGTTCAA; reverse, GCCGTCCTTGCT TAGTGATGA. GATA-3 forward, GACCCGAAACCGGAAG ATGT; reverse, CGCGTCATGCACCTTTT. RORrt forward, TGCGACTGGAGGACCTTCTAC; reverse, TCACCTCCT CCCGTGAAAAG. CD80 forward, TGGGAAAAACCCCC AGAAG; reverse, CCCCAAAGAGCACAAGTGTGT. MHCII forward, ACAGCCCAATGTCGTCATCTC; reverse, CCAG AGTGTTGTGGTGGTTGA. PD-L1 forward, CAGGCCGA GGGTTATCCA; reverse, CGGGTTGGTGGTCACTGTTT. CD74 forward, CCAACGCGACCTCATCTCTAA; reverse, AGGGCGGTTGCCCAGTA. CD86 forward, CTGTGGCC CTCCTCCTTGT; reverse, CTGATTCGGCTTCTTGTGAC ATA. IFN- $\gamma$ forward, TTGGCTTTGCAGCTCTTCCT; reverse, TGACTGTGCCGTGGCAGTA. TGF- $\beta$ forward,
GCAGTGGCTGAACCAAGGA; reverse, AGCAGTGAGCG CTGAATCG. IL-10 forward, GATGCCCCAGGCAGAGAA; reverse, CACCCAGGGAATTCAAATGC. IL-2 forward, GCAGGCCACAGAATTGAAAGA; reverse, TGCCGCAG AGGTCCAAGT.

Immunoblotting. Cell lysates were prepared in 2X LSB. Anti-PD-L1 antibody, anti-phospho-AKT (S308), anti-AKT, anti-phospho-S6 (T389), anti-S6K were purchased from Abcam. Anti- $\beta$-actin was purchased from Cell Signaling Technology.

CFSE staining. Cells were washed and resuspended in $5 \mu \mathrm{M}$ CellTrace CFSE dilution buffer, and stained for $15 \mathrm{~min}$ at $37^{\circ} \mathrm{C}$ in the dark. Cells were then centrifuged and washed in PBS containing $2 \%$ FBS twice.

Primary cell culture. The bone marrow-derived macrophages (BMDM) were prepared as follows: bone marrow cells were fushed from the femurs and tibias of C57BL/6 mice. The cells were then cultured at $2 \times 10^{6}$ cells per well in 24 -well plates in DMEM supplemented with $20 \mathrm{ng} / \mathrm{ml}$ murine M-CSF. Non-adherent cells were carefully removed, and fresh medium was added every 2 days. On day 6 , the cells were collected for experiments. Naive $\mathrm{CD}^{+} \mathrm{T}$ cells were enriched from splenic mononuclear cells by magnetic cell sorting using a mouse $\mathrm{CD}^{+}{ }^{+} \mathrm{T}$-cell isolation kit (Miltenyi Biotec). $\mathrm{T}$ cells were cultured with macrophages at a ratio of 5:1 in the presence of OVA peptide (Sigma), and the supernatants and cells were analyzed on day 3 of culture.

$\left[{ }^{3} \mathrm{H}\right]$ proliferation analysis. $\mathrm{CD} 4^{+} \mathrm{T}$ cell proliferation was performed as described (16). In brief, $\mathrm{CD}^{+} \mathrm{T}$ cells were cocultured with macrophages as described in 96-well plates for $72 \mathrm{~h}$. Proliferation was assessed based on the incorporation of $\left[{ }^{3} \mathrm{H}\right]$-thymidine $(1 \mu \mathrm{Ci} /$ well $)$ during the last $12 \mathrm{~h}$ of culture in triplicate wells. Cells were collected using a cell harvester, and $\left[{ }^{3} \mathrm{H}\right]$-thymidine was quantified using a scintillation counter.

T cell suppression assay. Primed-T cells were purified from BMDMs-T cell mixtures by flow cytometry using anti-CD25 and anti-Foxp3 antibodies. The purified $\mathrm{T}$ cells were co-cultured with CFSE-labeled activated $\mathrm{CD}^{+} \mathrm{T}$ cells at 1:1 ratio (primed T:CFSE-labeled T cell) in 96-well round bottom plates for an additional 3 days. CFSE intensity was monitored by flow cytometry.

Statistical analysis. Data in bar graphs are presented as mean \pm SEM. Differences between groups were analyzed with unpaired Student's t-tests. Statistical significance was set at $\mathrm{P}<0.05$.

\section{Results}

H37Rv infection promotes tumor growth in a mouse model. To determine the effect of H37Rv infection on the growth of non-small cell lung cancer (NSCLC), LLC cells were used to establish a tumor xenograft mouse model. We infected tumor-bearing mice with H37Rv at the same time. Results showed that the tumor volumes were clearly increased in 
A

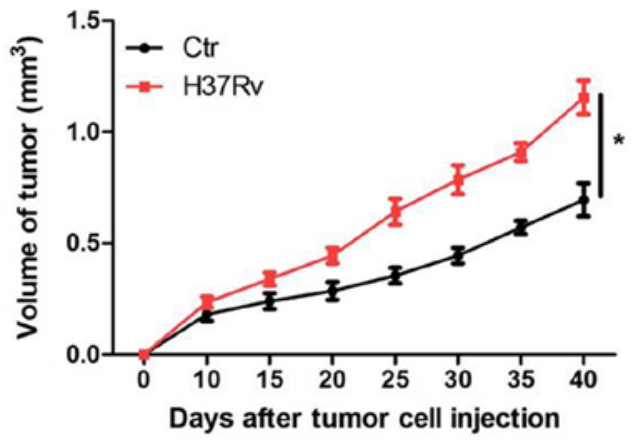

C
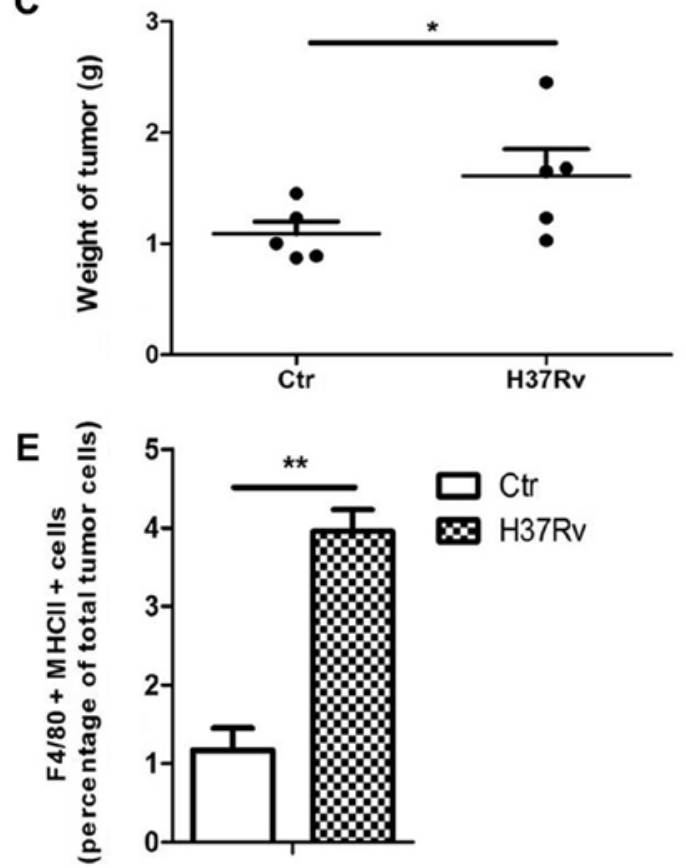

B

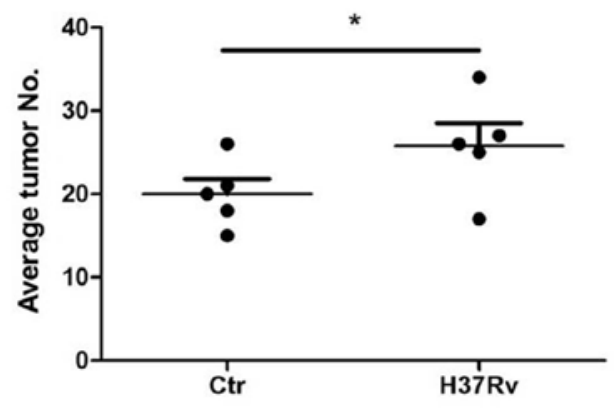

D

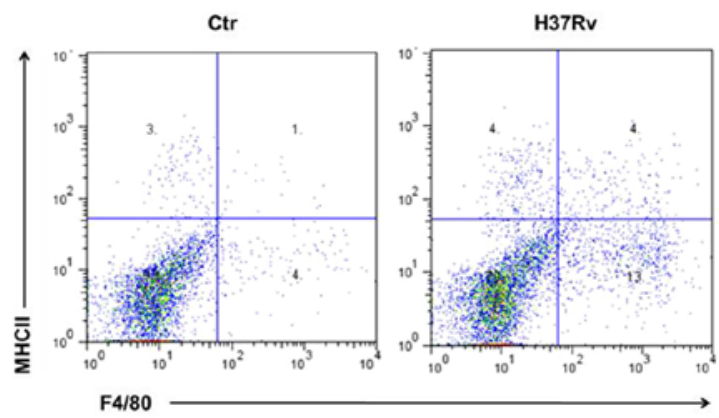

$\mathbf{F}$

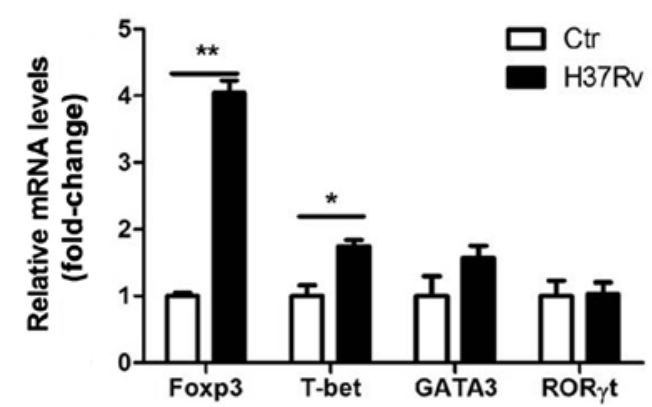

Figure 1. H37Rv infection promotes tumor growth in a mouse model. (A) Tumor growth was measured over time (n=10). (B) Number of lung tumor nodules in mice 40 days after intravenous tumor inoculation $(n=10)$. (C) Tumor mass 40 days after tumor cell inoculation $(\mathrm{n}=10)$. (D and $\mathrm{E}) \mathrm{F} 4 / 80^{+} \mathrm{MHCII}{ }^{+}$ tumor-associated macrophages were detected by flow cytometry. $(\mathrm{F}) \mathrm{CD} 4^{+} \mathrm{T}$ cell changes in the draining lymph nodes after H37Rv infection. All experiments were representative of three similar results. Statistical differences between groups are indicated by the $\mathrm{P}$-values. ${ }^{*} \mathrm{P}<0.05 ;{ }^{* *} \mathrm{P}<0.01$.

the H37Rv-infection group compared to the non-infection group (Fig. 1A). The number of tumors and tumor weight were significantly increased in the H37Rv-infection group as compared with the non-infection group (Fig. 1B and C). Macrophages are the main cell type infected by H37Rv. Macrophages sense the pathogen, produce cytokines and activate lymphocytes, thus restraining or promoting tumor development. We then assessed the changes of immune responses in the draining lymph nodes after $\mathrm{H} 37 \mathrm{Rv}$ infection. H37Rv infection induced tumor-associated macrophage activation as assessed by $\mathrm{F} 4 / 80^{+} \mathrm{MHCII}^{+}$by flow cytometry (Fig. 1D and E). Besides, H37Rv infection promoted Treg proportion with modest changes in other $\mathrm{T}$ cells types in the drainning lymph nodes (Fig. 1F). These results suggest that $\mathrm{H} 37 \mathrm{Rv}$ infection promotes tumor growth possibly through increasing Treg proportion.

H37Rv-infected macrophages promote Treg cell differentiation. To determine how H37Rv infection promoted Treg polarization, we coculture $\mathrm{H} 37 \mathrm{Rv}$-infected BMDMs with naive $\mathrm{CD}^{+} \mathrm{T}$ cells in vitro for $96 \mathrm{~h}$ as described (Fig. 2A). Of the cultured $\mathrm{BMDM}>80 \%$ are $\mathrm{F} 4 / 80^{+}$cells (data not shown). Flow cytometry analysis showed Treg was enhanced significantly when co-cultured with H37Rv-infected macrophages, as $\mathrm{CD}_{25} 5^{+} \mathrm{Foxp}^{+}$cell-percentage showed $>2$-fold increase (Fig. 2B and C). Generated Treg cells were also evaluated with a functional assay of $\mathrm{T}$ cell proliferation. We then purified the $\mathrm{CD} 25^{+} \mathrm{Foxp} 3^{+} \mathrm{T}$ cells from the co-culture system and further co-culture with CFSE-labeled activated CD $4^{+} \mathrm{T}$ cells for 3 days (Fig. $2 \mathrm{~A}$ ). Naïve $\mathrm{T}$ cells primed by H37Rv-infected macrophges had a stronger inhibitory effect on anti-CD3 and anti-CD28-stimulated T cells (Fig. 2C). The inhibitory effect was also observed when similar proliferation was studied by $\left[{ }^{3} \mathrm{H}\right]$-thymidine incorporation (Fig. 2D). These results suggest that $\mathrm{H} 37 \mathrm{Rv}$-infected macrophages promoted Treg cell differentiation.

H37Rv promotes macrophage activation and PD-L1 expression. Macrophage activation is necessary for priming and polarization of $\mathrm{T}$ cells. We found that macrophages were 
A

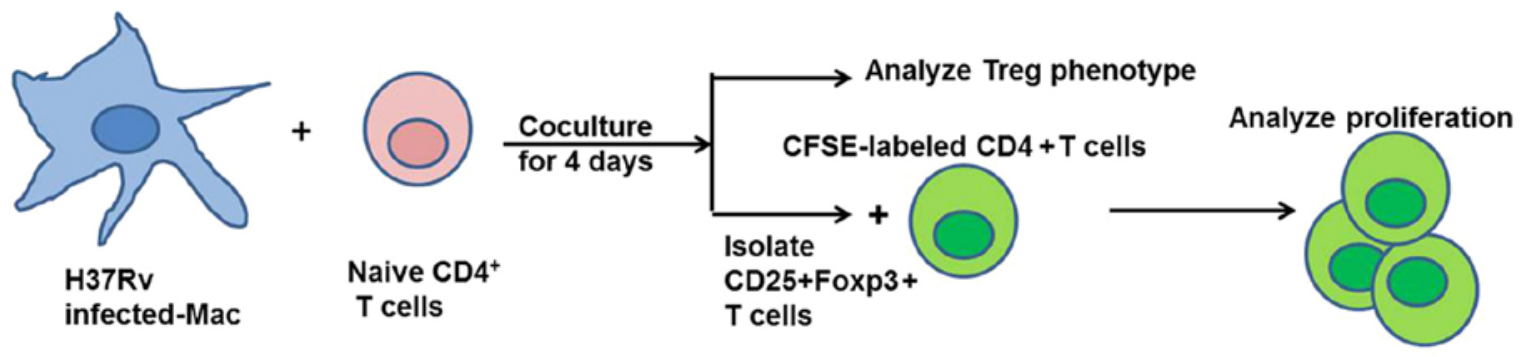

B

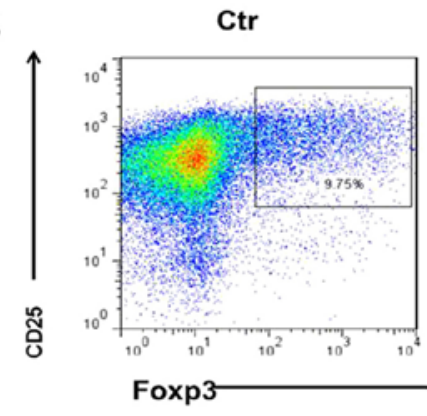

H37Rv

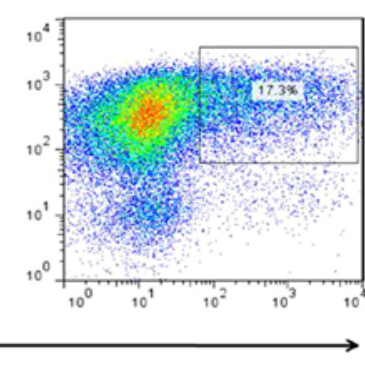

C

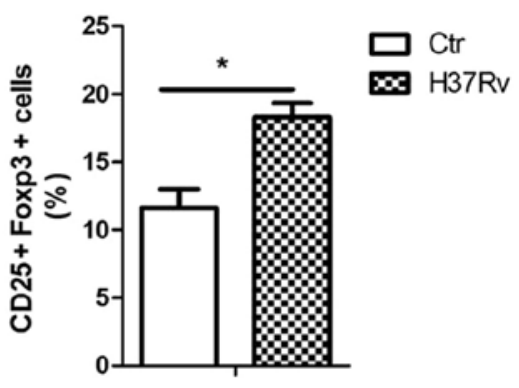

E

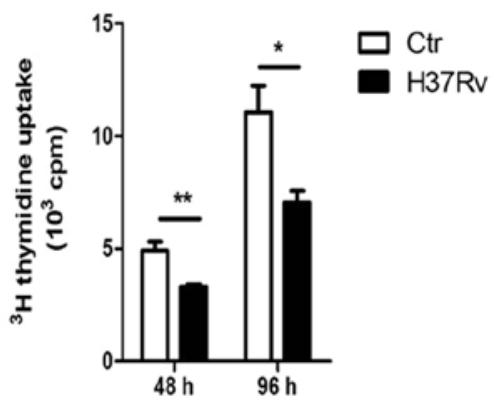

Figure 2. H37Rv-infected macrophages promote Treg cell differentiation. (A) Scheme of the Treg cell differentiation and suppression assay. Immature macrophages were treated with $\mathrm{H} 37 \mathrm{Rv}$ and co-cultured with $\mathrm{CD} 4^{+}$naïve $\mathrm{T}$ cells for 4 days. T cell populations were purified by using anti-CD3 specific antibody coated microbeads. (B and C) The Treg cell phenotype was detected by immunostaining with CD25 and Foxp3. (D) Functional assay of Treg cells for suppressing T cell proliferation. T cells primed by H37Rv-infected BMDMs were co-cultured with CFSE-labeled activated CD4 ${ }^{+} \mathrm{T}$ cells for 3 days. (E) Cells were co-cultured as in (D) and proliferation was analyzed by $\left[{ }^{3} \mathrm{H}\right]$-thymidine incorporation. Data are presented as $\mathrm{cpm}$. All experiments are representative of three similar results. Statistical differences between groups are indicated by the $\mathrm{P}$-values. ${ }^{*} \mathrm{P}<0.05 ;{ }^{* *} \mathrm{P}<0.01$.

activated upon $\mathrm{H} 37 \mathrm{Rv}$ stimulation. The surface expression of activation markers MHCII and CD80 was increased after H37Rv stimulation (Fig. 3A). In addition, their mRNA levels as well as CD74 and CD86, which both are activation markers, were all upregulated upon H37Rv infection (Fig. 3B). Treg cell function is controlled by many signaling pathways, including the IL-2, TGF- $\beta$ and PD-L1 mediated pathway. H37Rv-infected macrophages showed no increased IL-2 or TGF- $\beta$ expression (Fig. 3 C). However, the surface expression of PD-L1 was markedly increased (Fig. 3D and E). The total protein levels of PD-L1 were also upregulated after H37Rv infection, with a time-dependent manner (Fig. 3F). Blocking PD-L1 using anti-PD-L1 neutralizing antibody significantly impaired Treg proportion in the coculture system (Fig. 3G).

mTORCl activation contributes to the PD-L1 expression by $H 37 R v$ infection. PD-L1 expression was upregulated by a group of pro-inflammatory factors such as IL- 6 and TNF- $\alpha$. Also, PD-L1 expression can be downregulated by PTEN (17), which could inactivate the mTORC1 signal. Here, we showed that H37Rv significantly promoted PD-L1 expression with the elevated Akt and S6K phosphorylation, which both indicated activation of Akt-mTORC1 signal (Fig. 4).

Inhibition of mTORC1 signal attenuates PD-L1 expression induced by H37Rv stimulation. We then tried to determine whether mTORC1 inhibition will block PD-L1 expression by adopting mTORC1 inhibitor rapamycin. Rapamycin pre-treatment obviously attenuated PD-L1 expression upon H37Rv infection (Fig. 5A and B). Raptor is a specific component protein of mTORC1 while rictor is a specific component of mTORC2. Knock down of raptor, but not rictor, significantly lowered mTORC1 activity indicated by S6K phosphorylation (Fig. 5C and D). Consistently, raptor, but not rictor, deficiency decreased PD-L1 expression upregulated by H37Rv stimulation (Fig. 5C and D). Moreover, the Treg promotion ability of macrophages infected by H37Rv was also reduced with raptor deficiency (Fig. 5E).

mTOR inhibition decreases the ability of H37Rv infection in tumor acceleration. Considering the critical role of mTORC1 signal in the PD-L1 promotion and further Treg polarization, 
A
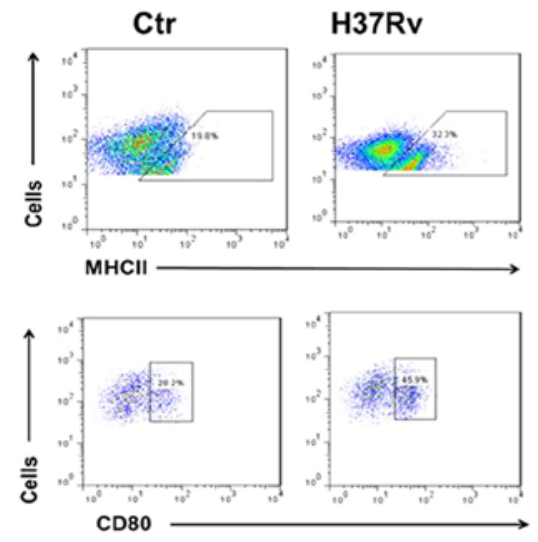

C

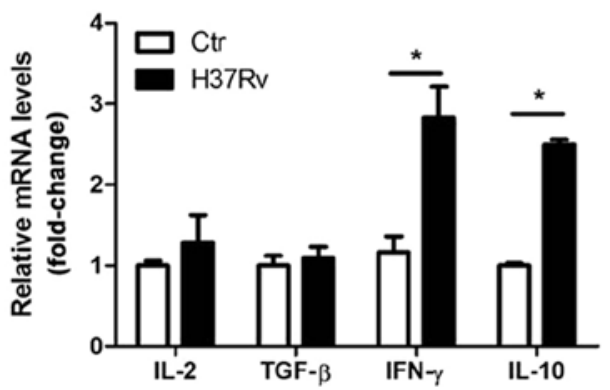

F

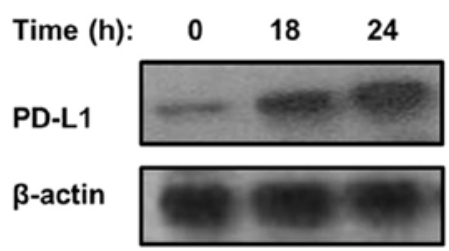

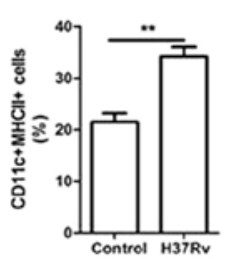

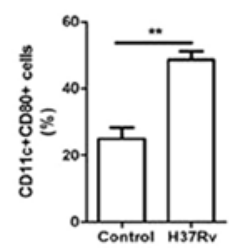

D

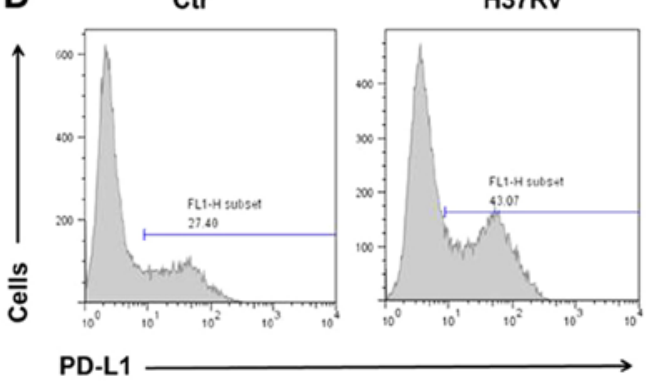

B

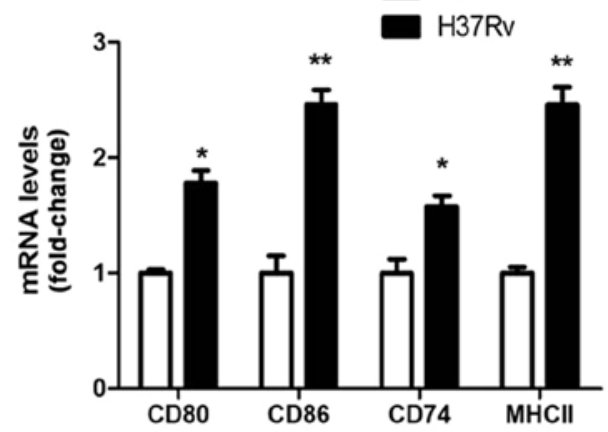

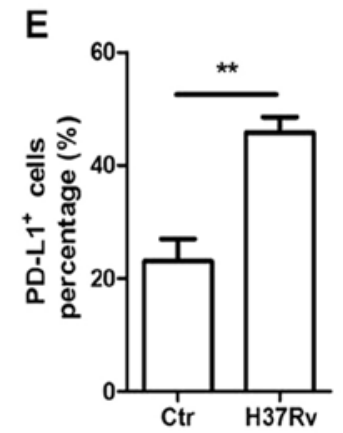

G

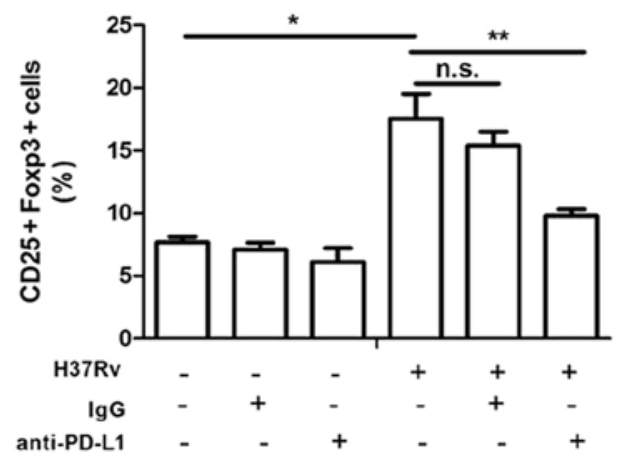

Figure 3. H37Rv promotes macrophage activation and PD-L1 expression. (A) Macrophage activation markers MHCII and CD80 were tested by FACS analysis after H37Rv stimulation. (B) mRNA levels of CD80, CD86, MHCII and CD74 were determined by RT-PCR. (C) mRNA levels of IL-2, TGF- $\beta$, IFN- $\gamma$ and IL-10 were determined by RT-PCR. (D and E) The surface expression of PD-L1 was analyzed by FACS. (F) Immunoblot analysis of total PD-L1 protein levels after H37Rv expression. (G) T cells were co-cultured with H37Rv-infected BMDMs in the presence of neutralizing antibody anti-PD-L1. CD25 ${ }^{+}$Foxp3 ${ }^{+}$ Tregs gated on CD4 were analyzed by flow cytometry. All experiments were representative of three similar results. Statistical differences between groups are indicated by the P-values. ${ }^{*} \mathrm{P}<0.05 ;{ }^{*} \mathrm{P}<0.01$.

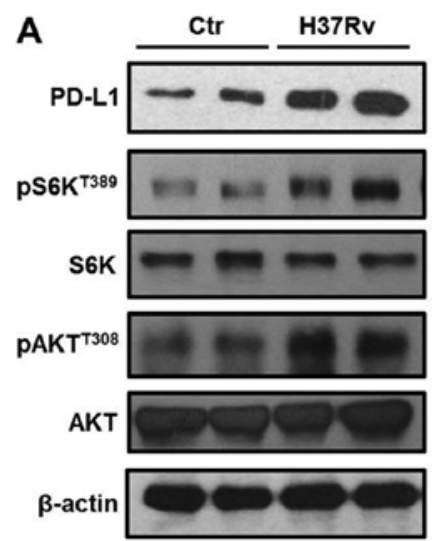

B

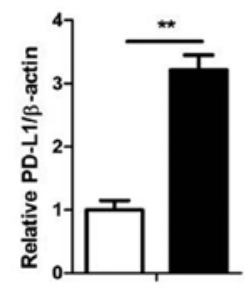

$\square \mathrm{Ctr}$
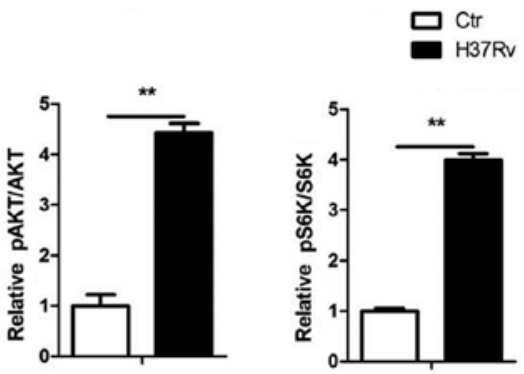

Figure 4. mTORC1 activation contributes to the PD-L1 expression by H37Rv infection. (A) H37Rv stimulated macrophages were collected and processed for immunoblotting. (B) Quantification of PD-L1, phophorylated S6K and Akt expression in (A). All experiments are representative of three similar results. Statistical differences between groups are indicated by the $\mathrm{P}$-values. ${ }^{*} \mathrm{P}<0.05 ;{ }^{* *} \mathrm{P}<0.01$. 
A
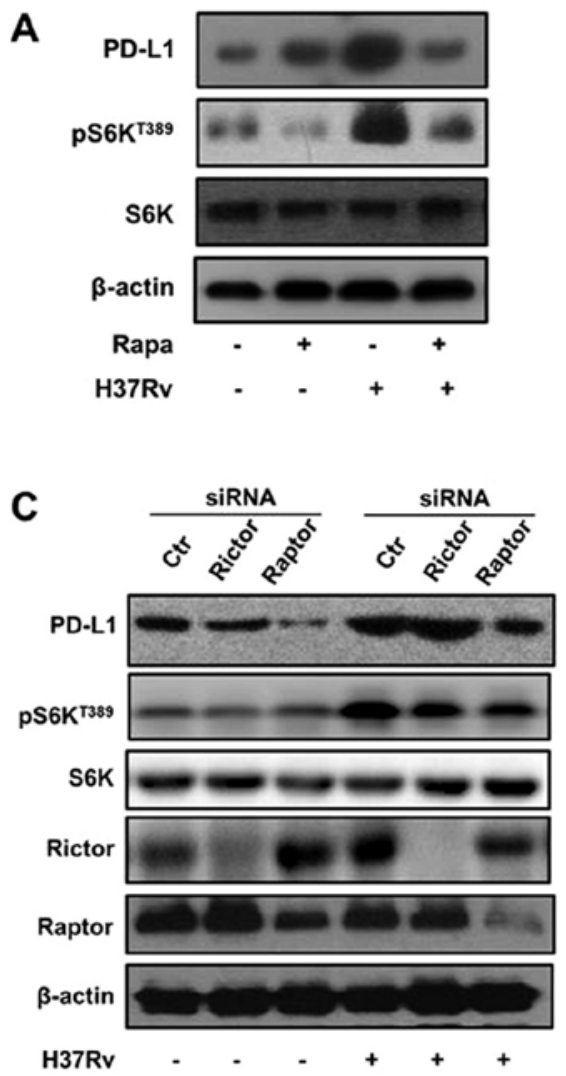

B

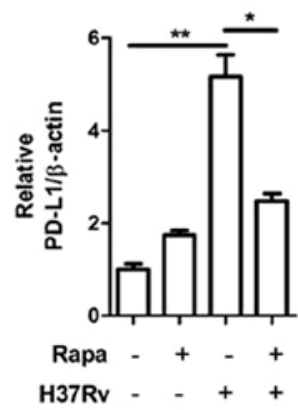

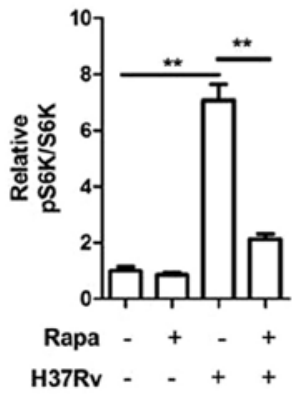

D

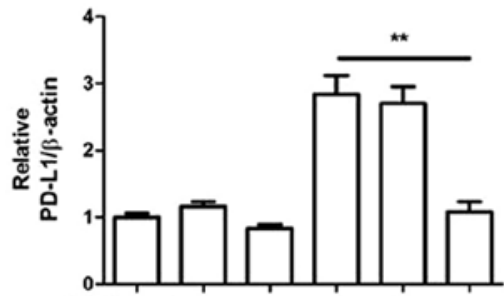

shRNA Ctr Rictor Raptor Ctr Rictor Raptor H37Rv

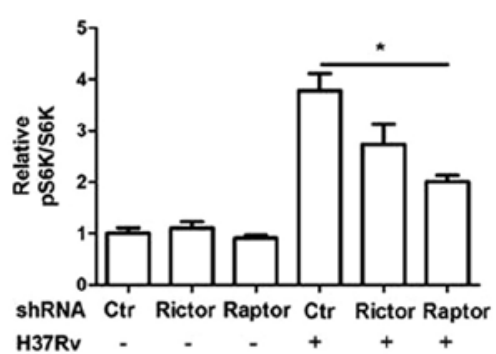

$\mathbf{F}$

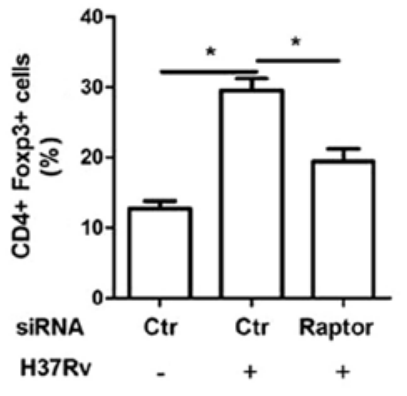

Figure 5. Inhibition of mTORC1 signal attenuated PD-L1 expression induced by H37Rv stimulation. BMDMs were pre-treated with rapamycin for 30 min and then treated with H37Rv or not. (A) The expression of PD-L1 expression is shown. (B) Quantification of PD-L1, phophorylated S6K in (A). (C) BMDMs were transfected with raptor or rictor siRNAs. The effects of raptor or rictor deficiency on PD-L1 expression upon H37Rv infection are shown by western blotting. (D) Quantification of PD-L1, phophorylated S6K in (C). (E and F) BMDMs treated as in (C) were co-cultured with naive CD4 ${ }^{+} \mathrm{T}$ cells for 3 days. The Treg cell phenotype were then analyzed by flow cytometry. All experiments are representative of three similar results. Statistical differences between groups are indicated by the $\mathrm{P}$-values. ${ }^{*} \mathrm{P}<0.05 ;{ }^{* *} \mathrm{P}<0.01$.

we investigated whether inhibition of mTORC1 could rescue the results of $\mathrm{H} 37 \mathrm{Rv}$ infection in tumor progression. We infected tumor-bearing mice with $\mathrm{H} 37 \mathrm{Rv}$ with or without rapamycin once a week. Tumor volumes were remarkably suppressed in the rapamycin-treatment group compared to the non-treatment group after H37Rv-infection, with comparable levels of non-infection groups (Fig. 6A). Besides, the tumor numbers and tumor weights were also decreased in the group treated with $\mathrm{H} 37 \mathrm{Rv}$ plus rapamycin, as compared with the H37Rv-infection group (Fig. 6B and C). Rapamycin treatment alone had a modest effect on tumor volumes, tumor weights and numbers, but without significant differences (Fig. 6A-C). Accordingly, additional rapamycin treatment blocked Treg proportion upon H37Rv infection (Fig. 6D). These results suggest that mTOCR1 is responsible for tumor acceleration and Treg promotion after $\mathrm{H} 37 \mathrm{Rv}$ infection.

\section{Discussion}

As one of the major antigen presenting cells (APC), macrophage activation delivers costimulation signals that lead to the activation of $\mathrm{CD}^{+} \mathrm{T}$ cells. $\mathrm{CD} 4^{+}$effector T cells are a heterogeneous population that can be divided into multiple subsets defined by their cytokine profiles. Th1 cells, which express the master transcription factor T-bet and secrete IFN- $\gamma$, is responsible for anti-bacteral and antitumor immunity $(18,19)$. Tregs, express high levels of CD25 and the transcription factor Foxp3, restrain immune responses including antitumor immunity (20-22). The 

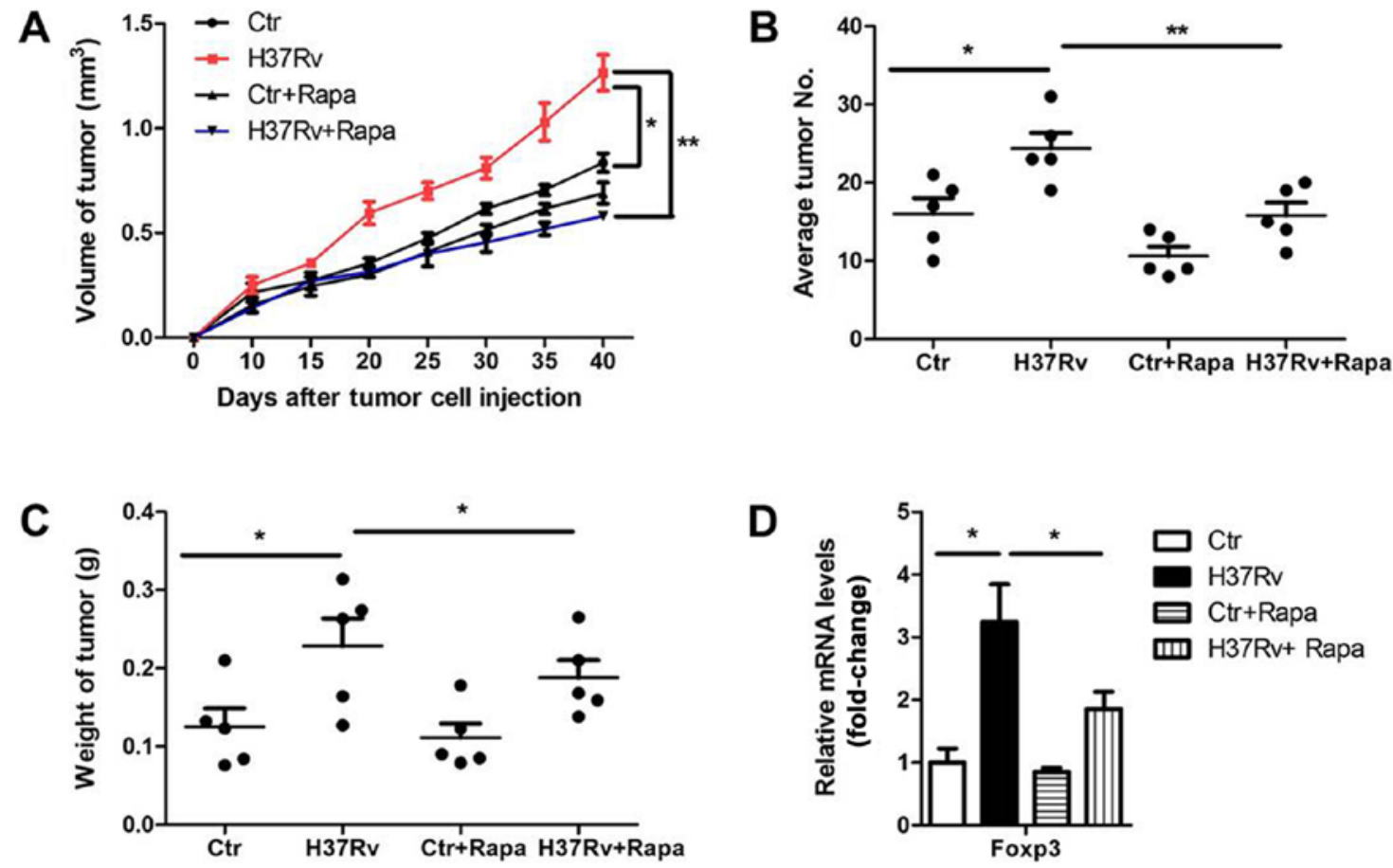

Figure 6. mTOR inhibition decreases the ability of H37Rv infection in tumor acceleration. Tumor-bearing mice were stimulated with H37Rv. At the same time, $4 \mathrm{mg} / \mathrm{kg}$ rapamycin or vehicle solution was injected intraperitoneally twice a week. (A) Tumor growth was measured over time (n=10). (B) Number of lung tumor nodules in mice 40 days after intravenous tumor inoculation $(n=10)$. (C) Tumor mass 40 days after tumor cell inoculation ( $\mathrm{n}=10)$. (D) Foxp3 mRNA levels in the draining lymph nodes after H37Rv plus rapamycin injection on day 40. All experiments are representative of three similar results. Statistical differences between the groups are indicated by the $\mathrm{P}$-values. ${ }^{*} \mathrm{P}<0.05 ;{ }^{* *} \mathrm{P}<0.01$.

tumor microenvironment is potently immunosuppressive as a result of inability of effector Th1 cell infiltration and suppressive Treg accumulation. Therefore, the immunoadjuvant component is needed to create a proinflammatory milieu, thereby enhancing costimulation and $\mathrm{T}$ lymphocyte activation (23). The ability of various infections to affect tumor growth has been well determined $(24,25)$. Immune adjuvants, such as tuberculosis Bacillus Calmette-Guérin (BCG) and heat-killed Mycobacterium vaccae suspension (SRL172), have been used as non-specific immunostimulators against various human cancers with suboptimal clinical response (26-28).

In this study, we intended to determine whether MTB infection could initiate effective antitumor immune responses. We first successfully infected mice with H37Rv (data not shown). Surprisingly, we found that H37Rv infection did not improve but promoted tumor development. We detected obvious Th1 cytokine profile that leads to an immune response against tumor cells in macrophges after $\mathrm{H} 37 \mathrm{Rv}$ stimulation. In the in vitro coculture system, Th1 and Treg proportion both were enhanced with a high Treg/Th1 ratio after H37Rv infection, which might to some extent explain the acceleration of tumor progression. Indeed, in Mycobacterium tuberculosis infection, Tregs proliferate and accumulate at sites of infection and prevent bacillary clearance in mice $(29,30)$. MTB promotes regulatory T-cell expansion via induction of PD-L1 (31-33). Depletion of Tregs enhances $M$. tuberculosis-induced IFN- $\gamma$ production by peripheral blood mononuclear cells (PBMCs) $(9,34)$.

Although macrophages showed upregulated MHCII, CD80 and CD86 expression upon H37Rv infection, the levels of PD-L1, which could interacts with the immune receptor programmed cell death-1 (PD-1) stimulated Foxp3 expression and Treg differentiation (35), was also markedly enhanced upon H37Rv stimulation, reflecting a negtive feed back mechanism to restrain robust inflammation. In fact, a direct correlation between PD-1 and IFN- $\gamma$ expression on NK cells was observed upon MTB infection (33). PD-L1 transcription is regulated by c-Jun and Stat3 (36). Akt-mTORC1 signal which is upstream of Stat3 has been reported to affect PD-L1 expression in various cells. Loss of Lkb1 and Pten leads to lung squamous cell carcinoma with elevated PD-L1 expression (17). In a human-mouse chimeric model of allograft rejection, rapamycin pretreatment of human arterial allografts increased graft endothelial cells expression of PD-L1 and PD-L2 and reduced subsequent infiltration of allogeneic effector $\mathrm{T}$ cells into the artery intima and intimal expansion (37). Oncogenic activation of the AKT-mTOR pathway promotes immune escape by driving expression of PD-L1 in syngeneic and genetically engineered mouse models of lung cancer (38). In our study, we found that H37Rv infection stimulated mTORC1 activation. Deficiency of mTORC1 by rapamycin or knockdown of raptor obviously abolished PD-L1 expression induced by H37Rv. Rapamycin treatment also reversed the ability of $\mathrm{H} 37 \mathrm{Rv}$ in tumor promotion.

Taken together, our results further strengthen that mTORC1 in macrophages controls Treg promotion and for the first time pointed out that concomitant $\mathrm{H} 37 \mathrm{Rv}$ infection will accelerate NSCLC progression. This study provides new insights for clinical treatment of NSCLC.

\section{Acknowledgements}

This study was supported by Science and Technology Commission guidance of Shanghai (no. 124119a6300); International Science 
and Technology Cooperation of Shanghai (no. 14430723300); National Natural Science Foundation of China (no. 81472642); 2014YZDC20700; Shanghai Municipal Education CommisionGaofeng Clininal Medicine Grant Support (no. 20161434); YZ2015-ZX12.

\section{References}

1. Paleiron N, Bylicki O, André M, Rivière E, Grassin F, Robinet G and Chouaïd C: Targeted therapy for localized non-small-cell lung cancer: A review. Onco Targets Ther 9: 4099-4104, 2016.

2. Whiteman DC and Wilson LF: The fractions of cancer attributable to modifiable factors: A global review. Cancer Epidemiol 44: 203-221, 2016.

3. Hamilton G, Rath B and Ulsperger E: A review of the role of surgery for small cell lung cancer and the potential prognostic value of enumeration of circulating tumor cells. Eur J Surg Oncol 42: 1296-1302, 2016.

4. Kuribayashi K, Funaguchi $\mathrm{N}$ and Nakano T: Chemotherapy for advanced non-small cell lung cancer with a focus on squamous cell carcinoma. J Cancer Res Ther 12: 528-534, 2016.

5. Giaj-Levra N, Ricchetti F and Alongi F: What is changing in radiotherapy for the treatment of locally advanced nonsmall cel lung cancer patients? A review. Cancer Invest 34: 80-93, 2016.

6. Chen L, He Z, Qin L, Li Q, Shi X, Zhao S, Chen L, Zhong N and Chen X: Antitumor effect of malaria parasite infection in a murine Lewis lung cancer model through induction of innate and adaptive immunity. PLoS One 6: e24407, 2011.

7. Kim JO, Jung SS, Kim SY, Kim TY, Shin DW, Lee JH and Lee YH: Inhibition of Lewis lung carcinoma growth by Toxoplasma gondii through induction of Th1 immune responses and inhibition of angiogenesis. J Korean Med Sci 22 (Suppl) S38-S46, 2007.

8. Martínez A, Torello $\mathrm{S}$ and Kolter R: Sliding motility in mycobacteria. J Bacteriol 181: 7331-7338, 1999.

9. Garg A, Barnes PF, Roy S, Quiroga MF, Wu S, García VE, Krutzik SR, Weis SE and Vankayalapati R: Mannose-capped lipoarabinomannan- and prostaglandin E2-dependent expansion of regulatory T cells in human Mycobacterium tuberculosis infection. Eur J Immunol 38: 459-469, 2008.

10. Gehring AJ, Rojas RE, Canaday DH, Lakey DL, Harding CV and Boom WH: The Mycobacterium tuberculosis 19-kilodalton lipoprotein inhibits gamma interferon-regulated HLA-DR and $\mathrm{Fc}$ gamma R1 on human macrophages through Toll-like receptor 2. Infect Immun 71: 4487-4497, 2003.

11. Liu H, Komai-Koma M, Xu D and Liew FY: Toll-like receptor 2 signaling modulates the functions of $\mathrm{CD} 4^{+} \mathrm{CD} 25^{+}$regulatory T cells. Proc Natl Acad Sci USA 103: 7048-7053, 2006.

12. Hirsch CS, Rojas R, Wu M and Toossi Z: Mycobacterium tuberculosis induces expansion of Foxp3 positive CD4 T-cells with a regulatory profile in tuberculin non-sensitized healthy subjects: Implications for effective immunization against TB. J Clin Cell Immunol 7: 7, 2016.

13. He XY, Xiao L, Chen HB, Hao J, Li J, Wang YJ, He K, Gao Y and Shi BY: T regulatory cells and Th1/Th2 cytokines in peripheral blood from tuberculosis patients. Eur J Clin Microbiol Infect Dis 29: 643-650, 2010.

14. Kuo CH, Lo CY, Chung FT, Lee KY, Lin SM, Wang CH, Heh CC, Chen HC and Kuo HP: Concomitant active tuberculosis prolongs survival in non-small cell lung cancer: A study in a tuberculosisendemic country. PLoS One 7: e33226, 2012.

15. Chen C, Liu Y, Liu Y and Zheng P: mTOR regulation and therapeutic rejuvenation of aging hematopoietic stem cells. Sci Signal 2: ra75, 2009.

16. Zhou H, Wang Y, Lian Q, Yang B, Ma Y, Wu X, Sun S, Liu Y and Sun B: Differential IL-10 production by DCs determines the distinct adjuvant effects of LPS and PTX in EAE induction. Eur J Immunol 44: 1352-1362, 2014.

17. Xu C, Fillmore CM, Koyama S, Wu H, Zhao Y, Chen Z, HerterSprie GS, Akbay EA, Tchaicha JH, Altabef A, et al: Loss of Lkb1 and Pten leads to lung squamous cell carcinoma with elevated PD-L1 expression. Cancer Cell 25: 590-604, 2014.

18. Zamarron BF and Chen W: Dual roles of immune cells and their factors in cancer development and progression. Int J Biol Sci 7: 651-658, 2011.

19. Wieder T, Braumüller H, Kneilling M, Pichler B and Röcken M: T cell-mediated help against tumors. Cell Cycle 7: 2974-2977, 2008 .
20. Sakaguchi S, Miyara M, Costantino CM and Hafler DA: FOXP3 ${ }^{+}$ regulatory $\mathrm{T}$ cells in the human immune system. Nat Rev Immunol 10: 490-500, 2010.

21. Maynard CL, Hatton RD, Helms WS, Oliver JR, Stephensen CB and Weaver CT: Contrasting roles for all-trans retinoic acid in TGF-beta-mediated induction of Foxp3 and I110 genes in developing regulatory T cells. J Exp Med 206: 343-357, 2009.

22. Roychoudhuri R, Eil RL and Restifo NP: The interplay of effector and regulatory $\mathrm{T}$ cells in cancer. Curr Opin Immunol 33: 101-111, 2015.

23. Yasumoto K, Hanagiri T and Takenoyama M: Lung cancer-associated tumor antigens and the present status of immunotherapy against non-small-cell lung cancer. Gen Thorac Cardiovasc Surg 57: 449-457, 2009.

24. Alexandroff AB, Jackson AM, O'Donnell MA and James K: BCG immunotherapy of bladder cancer: 20 years on. Lancet 353 : 1689-1694, 1999.

25. Hibbs JB Jr, Lambert LH Jr and Remington JS: Resistance to murine tumors conferred by chronic infection with intracellular protozoa, Toxoplasma gondii and Besnoitia jellisoni. J Infect Dis 124: 587-592, 1971.

26. Grange JM, Bottasso O, Stanford CA and Stanford JL: The use of mycobacterial adjuvant-based agents for immunotherapy of cancer. Vaccine 26: 4984-4990, 2008.

27. Malmström PU, Wijkström H, Lundholm C, Wester K, Busch C and Norlén BJ; Swedish-Norwegian Bladder Cancer Study Group: 5-year followup of a randomized prospective study comparing mitomycin $\mathrm{C}$ and bacillus Calmette-Guerin in patients with superficial bladder carcinoma. J Urol 161: 1124-1127, 1999.

28. Stanford JL, Stanford CA, O'Brien ME and Grange JM: Successful immunotherapy with Mycobacterium vaccae in the treatment of adenocarcinoma of the lung. Eur J Cancer 44: 224-227, 2008.

29. Ordway D, Henao-Tamayo M, Harton M, Palanisamy G, Troudt J, Shanley C, Basaraba RJ and Orme IM: The hypervirulent Mycobacterium tuberculosis strain HN878 induces a potent TH1 response followed by rapid down-regulation. J Immunol 179: 522-531, 2007.

30. Scott-Browne JP, Shafiani S, Tucker-Heard G, Ishida-Tsubota K, Fontenot JD, Rudensky AY, Bevan MJ and Urdahl KB: Expansion and function of Foxp3-expressing T regulatory cells during tuberculosis. J Exp Med 204: 2159-2169, 2007.

31. Trinath J, Maddur MS, Kaveri SV, Balaji KN and Bayry J: Mycobacterium tuberculosis promotes regulatory T-cell expansion via induction of programmed death-1 ligand 1 (PD-L1, CD274) on dendritic cells. J Infect Dis 205: 694-696, 2012.

32. Periasamy S, Dhiman R, Barnes PF, Paidipally P, Tvinnereim A, Bandaru A, Valluri VL and Vankayalapati R: Programmed death 1 and cytokine inducible SH2-containing protein dependent expansion of regulatory T cells upon stimulation With Mycobacterium tuberculosis. J Infect Dis 203: 1256-1263, 2011.

33. Alvarez IB, Pasquinelli V, Jurado JO, Abbate E, Musella RM, de la Barrera SS and García VE: Role played by the programmed death-1-programmed death ligand pathway during innate immunity against Mycobacterium tuberculosis. J Infect Dis 202: 524-532, 2010.

34. Li L, Lao SH and $\mathrm{Wu} \mathrm{CY}$ : Increased frequency of CD4(+) CD25(high) Treg cells inhibit BCG-specific induction of IFN-gamma by CD4(+) T cells from TB patients. Tuberculosis (Edinb) 87: 526-534, 2007.

35. Francisco LM, Salinas VH, Brown KE, Vanguri VK, Freeman GJ, Kuchroo VK and Sharpe AH: PD-L1 regulates the development, maintenance, and function of induced regulatory T cells. J Exp Med 206: 3015-3029, 2009.

36. Jiang X, Zhou J, Giobbie-Hurder A, Wargo J and Hodi FS: The activation of MAPK in melanoma cells resistant to BRAF inhibition promotes PD-L1 expression that is reversible by MEK and PI3K inhibition. Clin Cancer Res 19: 598-609, 2013.

37. Wang C, Yi T, Qin L, Maldonado RA, von Andrian UH, Kulkarni S, Tellides G and Pober JS: Rapamycin-treated human endothelial cells preferentially activate allogeneic regulatory T cells. J Clin Invest 123: 1677-1693, 2013.

38. Lastwika KJ, Wilson W III, Li QK, Norris J, Xu H, Ghazarian SR, Kitagawa H, Kawabata S, Taube JM, Yao S, et al: Control of PD-L1 expression by oncogenic activation of the AKT-mTOR pathway in non-small cell lung cancer. Cancer Res 76: 227-238, 2016. 